\title{
Téoros
}

Revue de recherche en tourisme

\section{Le bon ménage peut-il durer ?}

\section{Marc Laplante}

Volume 6, numéro 1, mars 1987

Tourisme et transports

URI : https://id.erudit.org/iderudit/1080516ar

DOI : https://doi.org/10.7202/1080516ar

Aller au sommaire du numéro

Éditeur(s)

Université du Québec à Montréal

ISSN

0712-8657 (imprimé)

1923-2705 (numérique)

Découvrir la revue

Citer ce document

Laplante, M. (1987). Le bon ménage peut-il durer ? Téoros, 6(1), 2-3.

https://doi.org/10.7202/1080516ar

Ce document est protégé par la loi sur le droit d'auteur. L'utilisation des services d'Érudit (y compris la reproduction) est assujettie à sa politique d'utilisation que vous pouvez consulter en ligne.

https://apropos.erudit.org/fr/usagers/politique-dutilisation/
Cet article est diffusé et préservé par Érudit.

Érudit est un consortium interuniversitaire sans but lucratif composé de l'Université de Montréal, l'Université Laval et l'Université du Québec à Montréal. Il a pour mission la promotion et la valorisation de la recherche. https://www.erudit.org/fr/ 


\section{Présentation}

\section{Le tourisme et les transports}

La plupart du temps, le développement de l'industrie touristique est le fidèle reflet du développement de l'industrie des transports. A certains moments, mais rarement, c'est la demande touris tique qui mène le jeu; à d'autres moments, et le plus souvent, ce sont les nouvelles technologies des transports qui vont conditionner la demande touristique.

Ces remarques sont valables pour le Québec et ses voisins et dans I'optique où plusieurs modes de transport pourraient ètre concurrentiels. En considérant /'évolution des liens entre le tourisme et le transport on a l'impression que le transport forme l'infrastructure du système et le tourisme la superstructure.

Nous savons que dans la pensée marxiste ce sont les infrastructures qui dominent et imposent des solutions aux différents problèmes socio-économiques. Dans cette dialectique constante, le tourisme perçu comme culture, comme modèle, et même comme style de vie, est de plus en plus réduit à la portion congrue. Ce sont les impératifs technologiques qui dictent la forme des relations entre l'industrie du tourisme et celle des transports.

A la réflexion, cette domination n'a rien d'étonnant, rindustrie des transports, pour survive, accorde un röle déterminant au progrès technique, à l'efficacité des movens de production par le biais de constantes innovations, elle engage dans ce processus d'énormes investissements, pour elle c'est une question de vie ou de mort.

L'industrie touristique, au sens le plus large du terme, est un secteur mou de l'économie oủ les investissements sont faibles et ouे, habituellement, on accorde peu d'importance aux découvertes technologiques et au développement du "capital humain". Dans cette perspective, le tourisme peut difficilement résister aux ajustements constants imposés par le systeme des transports.

Cette domination n'est peut-être pas inéluctable et éternelle mais elle pèse actuellement d'un poids énorme sur toutes les stratégies de développement et de planification (surtout en milieu régional) touristiques.

La forte concurrence entre les moyens de transport tend à une réduction quasi-systématique des choix: depuis 1940 , le bateau et le train sont presque disparus comme modes de transport touristique. L'automobile semble plafonner et seuls l'autobus et l'avion ont une croissance constante. Les contraintes telles que les coûts, le temps et la sécurité mènent à une limitation des possibilités.

La limitation du choix des moyens de transport entraine aussi des restrictions au niveau du choix des destinations; par exemple, la longueur des pistes d'atterrissage va devenir une condition préalable à la venue de touristes. Donc, la réduction des moyens de transport va amener une réduction de l'espace et la valorisation mécanique de corridors touristiques aux dépens des autres destinations.

La diversite et l'efficacité des modes de transport sont de bons indicateurs de la santé du tourisme. L'excès de spécialisation du système de transport est un danger mortel pour l'industrie touristique québécoise. C'est donc aux intervenants touristiques de proposer, d'agencer certaines alternatives qui favoriseraient une utilisation optimale de plusieurs modes de transports.

Ce pluralisme des moyens de transport est vital pour le tourisme régional. // est aussi nécessaire pour les grandes viles; quand leurs attraits s'usent et vieillissent elles doivent alors s'appuyer sur l'arrière pays pour redevenir attractives. D'une façon ou d'une autre, /'avenir du transport est aussi /'avenir du tourisme et les defaites de l'un seront les défaites de l'autre $f$

Jean Stafford

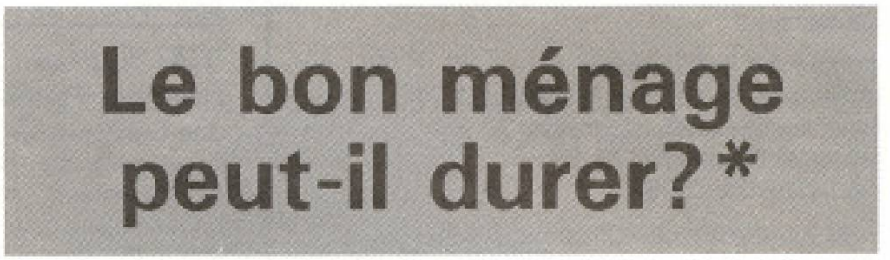

$\mathrm{Si}$, parce que les coûts augmentent, le touriste est amené à ménager ses transports, il doit encore et toujours se déplacer dans un espace géographique pour satisfaire la condition essentielle de l'expérience touristique. L'étude des moyens de transport reste donc un sujet privilégié pour traiter du tourisme.

En fait, nous pourrions écrire une bonne part de l'histoire et de l'évolution récente du tourisme en suivant les changements survenus dans les moyens de transport.

"Editorial collectif par l'equipe de rédaction de TÁoros: redigh par Mare Laplante, sociologue, Departement detudes urbaines, UQAM. 


\section{Le tourisme profite des révolutions technologiques}

Les révolutions technologiques furent presque toujours spectaculaires dans ce domaine car les enjeux des moyens de transport débordent largement ceux du tourisme et concernent tous les développements des sociétés industrielles. Le tourisme, en cette matière, fut le grand profiteur: il n'a fait naître aucun des moyens de transport modernes mais il fut souvent un des premiers secteurs à tirer profit de chaque invention. Ses bonds en avant sont lies aux performances des moyens de transport.

Des régions se sont ouvertes touristiquement à la suite d'une poussée des moyens de transport. L'histoire de la Côte d'Azur, par exemple, suit celle des avancées du train. La région touristique des Laurentides doit beaucoup au petit train du nord d'abord. à l'autoroute ensuite. Dès les années 1925-1930, les principaux guides touristiques du Québec sont structurés selon le réseau routier de l'époque, chaque route constituant un itinéraire détaillé. Ces guides d'ailleurs étaient produits par le ministère de la Voirie et destinés aux Américains. Ceux-ci vivaient alors l'époque glorieuse de l'automobile de plaisance et n'hésitaient pas à s'engager sur les périlleuses voies gaspésiennes pour un tour qui pouvait prendre de 10 à 20 jours.

Au cours des années plus récentes, des changements d'habitudes touristiques (et de valeurs) sont très liees aux performances des moyens de transport: ainsi, puisque l'avion permet maintenant d'atteindre des destinations éloignées en très peu de temps, le temps de déplacement semble ne plus faire partie de l'expérience touristique totale. A une autre epoque, l'aller et le retour sur une destination comptaient autant - sinon davantage - que la destination elle-même. Le journal typique d'un jeune aristocrate anglais faisant son Grand Tour s'attardait à décrire Paris, Madrid, Rome ou Vienne, mais l'essentiel portait sur les routes parcourues, les étapes, les relais, l'itinérance même, le fait d'être en mouvement, de voir constamment changer les paysages, les styles, les modes de vie, les us et coutumes, les règles sociales. etc... De nos jours, les voyageries ont perdu leur valeur au profit des destinations. Le tourisme de séjour, d'activités en une mềme localisation, est très lié à la rapidité du transport. Un modèle de prise de vacances qui s'impose de plus en plus consiste à se faire parachuter deux, trois ou quatre fois par année, sur une destination spécialisée pour se livrer là à ses activités favorites: plage, sports, arts, etc... Pour arriver à cela, il a fallu des transports trè rapides et sommes toutes, relativement peu coûteux; l'avion de masse, les charters, les vols nolisés avec de gros porteurs, les vols de nuit, etc., ont favorisé ces types de projets touristiques. Il y a une centaine d'années. le temps du déplacement était, en lui-même, un moment important de l'aventure d'un voyage et les services des Cook, des Pullman, etc., ont cherché à rendre agréable les heures et les jours passés dans les moyens de transport. Bientôt, par la puissance des nouvelles technologies, on pourra passer de l'Angleterre à la France sans s'en rendre compte - ou presque - grâce au tunnel sous la Manche. On parle aussi d"un train propulsé dans un tunnel qui relierait New York à Los Angeles en 2 heures!

\section{Des moyens de transport comme loisirs}

Alors que les temps de transport se racourcissent, d'anciens moyens de transport survivent ou retrouvent une popularité par le tourisme contemporain dont un des produits à la mode est justement le transport-activité: bateaux de croisières, train de croisière, mongolfiere, vélo ou cyclo-tourisme, tourisme hippique, péniches, voiliers, trecking, etc...

De même, d'autres installations et produits ont acquis une seconde vocation - spécifiquement touristique - après avoir servi d'abord de voie ou de moyen de transport: pensons aux anciennes route souvent rebaptisées voies "panoramiques" depuis la construction des autoroutes "aveugles", pensons aux anciennes gares qui ser- vent aujourd'hui de restaurants, de bureaux d'informations touristiques, de musées, etc.; pensons aussi à cet hébergement si typique: le motel, pensé d'abord pour les voyageurs en automobile et converti souvent en lieu de loisir pour la population locale!

Parmi les attractions touristiques offertes régulièrement aux visiteurs, on trouve plusieurs plaisirs associés aux transports: les gondoles à Venise, les bateaux-mouches sur la Seine, les canaux d'Amsterdam, avec les vélos; on trouve aussi de fréquentes invitations à partager les transports en communs des villes ou régions visitées, pour favoriser un contact avec les gens, leur vie quotidienne, etc. (activité dont plusieurs touristes se privent par peur. justement, de cotoyer les visités dans leur vie réelle).

Enfin, rappelons comment sont populaires aujourd'hui les forfaits comprenant un mix de transport: avion-auto, avion-croisière, aviontrain, auto-croisière, etc...

En vacances, on continue donc à jouer avec les moyens de transport: on se paiera huit heures en jet le plus rapide pour ensuitc s'installer dans l'Orient Express et refaire une partie de son célebre parcours; mieux encore, douze heures en 747 pour une expédition dans le désert à dos de chameau ou au pôle, en traîneau à chiens.

A une époque de très grande mobilité comme la nôtre, les bouleversements dans les moyens de transports n'ont pas que d'heureux effets. L'histoire des villes, par exemple, est en rapport ćtroit avec celle des transports: tel carrefour marchand au temps des carosses fut ensuite déclassé par les nouvelles jonctions des voies ferrovières, pour retrouver une vitalité avec l'arrivée de l'automobile et retomber dans l'ennui avec les réseaux aériens. Le déclin du che. min de fer a ruiné des destinations touristiques qui en dépendaient trop exclusivement.

Enfin, si on veut s'inquiéter vraiment, il faut s'attarder au fait que les moyens de transport actuels permettent quasiment d'effacer le temps de déplacement. Les espaces parcourus n'ont plus de réalité: du haut des airs, les nuages sont plus réels que le sol; les très longs parcours dans les autocars modernes ne font prendre contact avec la réalité qu'aux relais: le reste défile à toute allure de l'autre côté des vitres teintées; dans une aire donnnée, l'automobiliste emprunte les voles rapides pour aller d'une place à l'autre: le reste du pays n'existe plus; les vacances dans les stations touristiques ne comprennent souvent que de brèves sorties organisées dans le milieu environnant: comment saisir alors la géographie d'un tel pays? Comment comprendre le Québec dont certains villages sont à 1000 kilomètres de Montréal quand le visiteur débarque à Mirabel, passe deux jours à Montréal, s'aventure jusqu'à Québec par une autoroute, revient à Montréal par une autre et repart en avion vers Toronto? On ne compte plus les critiques dénonçant le touriste comme un "voyeur"! Pourtant, ce touriste se dit visiteur ce qui signifie - dans les dictionnaires au moins - qu'il parcoure avec attention le lieu visité.

Les moyens de transports ont façonné les sociétés modernes et donné au tourisme son dynamisme principal; reste à savoir s'ils auront autant de poids dans le tourisme de demain. $f$ 